\title{
Comportamento mecânico e clínico de próteses monolíticas à base de zircônia: revisão de literatura
}

\section{(Mechanical and clinical behavior of zirconia-based monolithic prosthesis: literature review)}

\author{
R. Ottoni ${ }^{1}$, M. Borba ${ }^{1}$ \\ ${ }^{1}$ Universidade de Passo Fundo, Programa de Pós-Graduação, Faculdade de Odontologia, BR286, 99052-900, \\ Passo Fundo, RS, Brasil
}

\begin{abstract}
Resumo
Próteses fixas com infraestrutura de metal ou cerâmica apresentam muitas falhas devido ao lascamento da cerâmica vítrea de cobertura. Assim, próteses monolíticas produzidas com apenas um material foram desenvolvidas no intuito de diminuir estas falhas. Cerâmicas à base de zircônia tiveram suas propriedades ópticas modificadas para permitir a confecção de próteses monolíticas. Portanto, o objetivo deste estudo foi verificar através de uma revisão da literatura o comportamento mecânico e clínico das próteses monolíticas à base de zircônia. Foi feita uma busca na base de dados PubMed/Medline (2010 a 2017) utilizando as palavras-chave: zircônia (zirconia), monolítica (monolithic) e prótese (prosthesis). Foram selecionados 13 estudos laboratoriais e 5 estudos clínicos. Os estudos laboratoriais mostraram que próteses monolíticas à base de zircônia apresentam alta resistência à fratura, à fadiga e ao lascamento. Próteses com espessura mínima de $0,7 \mathrm{~mm}$ devem ser confeccionadas para que sejam suficientemente resistentes às cargas impostas pela mastigação, tendo carga de fratura semelhante a coroas metalocerâmicas. Os estudos clínicos relatam baixas taxas de falha para esse tratamento protético. Próteses monolíticas à base de zircônia apresentam comportamento mecânico e clínico favorável para a reabilitação da região posterior.

Palavras-chave: cerâmicas, mecânica, prótese dentária.
\end{abstract}

\begin{abstract}
Dental prosthesis produced with metal or ceramic infrastructure show high rates of chipping of the glass-ceramic veneer. Thus, monolithic prosthetic restorations produced with only one material were developed in order to reduce these failures. Zirconia-based ceramics had their optical properties modified to produce monolithic restorations. Therefore, the objective of this study was to verify, through a literature review, the mechanical and clinical behavior of zirconia-based monolithic dental prostheses. PubMed/Medline database was used to search for manuscripts (2010 to 2017) using the following keywords: zirconia, monolithic and prosthesis. Thirteen in vitro studies and five clinical studies were selected. Studies have shown that zirconia-based monolithic prostheses exhibit high resistance to fracture, fatigue and chipping. To support the chewing load and achieve similar mechanical behavior as the metalceramic system, restorations should be produced with a minimum thickness of $0.7 \mathrm{~mm}$. Clinical studies report low failure rates for this prosthetic treatment. Zirconia-based monolithic dental prostheses present mechanical and clinical behavior favorable for the rehabilitation of the posterior region.
\end{abstract}

Keywords: ceramics, dental prosthesis, mechanics.

\section{INTRODUÇÃO}

Cerâmicas à base de zircônia foram inicialmente introduzidas na Odontologia com o objetivo de substituir a infraestrutura metálica das próteses fixas. A cerâmica policristalina de zircônia tetragonal estabilizada por óxido de ítrio (3 mol\% de ítria; 3Y-TZP) destaca-se por apresentar propriedades mecânicas superiores às demais cerâmicas odontológicas por causa do seu mecanismo de tenacificação por transformação de fase (transformation toughening). Quando a 3Y-TZP é submetida a tensões (ex.: mastigação, desgaste, jateamento de partículas, polimento) ocorre a transfor-

*marciaborba@upf.br mação da fase cristalina tetragonal para monoclínica. Essa transformação gera tensões compressivas que se opõem às tensões de tração que levariam à propagação dos defeitos, resultando em aumento da tenacidade e resistência à fratura do material. A resistência à flexão da 3 Y-TZP varia entre 840 e $1600 \mathrm{MPa}$, a tenacidade à fratura é de 5,9 a 7,4 MPa.m ${ }^{1 / 2}$, o módulo de elasticidade é de $220 \mathrm{GPa}$ e a dureza é de $12 \mathrm{GPa}$ [1-5]. Entretanto, a 3Y-TZP apresenta algumas limitações relacionadas às suas propriedades ópticas, sendo necessário o recobrimento da infraestrutura de 3Y-TZP com cerâmicas vítreas (ex.: porcelana feldspática) para alcançar a cor e translucidez da estrutura dental. Porém, essas próteses multicamadas são mais susceptíveis ao lascamento e à delaminação [6]. Coroas de 3Y-TZP apresentam taxa de falha anual 
de $1,84 \%$ e sobrevivência após cinco anos de $91,2 \%$, taxa de lascamento anual de $0,64 \%$ e após 5 anos de 3,1\% [7]. Próteses parciais fixas (PPFs) à base de zircônia apresentam taxa de falha anual de $2,02 \%$ e sobrevivência após cinco anos de $90,4 \%$, taxa de lascamento anual de $4,33 \%$ e após cinco anos de 19,5\%, ficando atrás apenas de descoloração marginal $(6,72 \%$ e $28,5 \%)$ [8]. Já outra avaliação encontrou taxa de falha de $0,71 \%$ para coroas unitárias e $2,6 \%$ para PPFs após 5 anos [9].

Durante a função clínica, as restaurações dentais são sujeitas a forças mastigatórias que podem variar entre $441 \mathrm{e}$ $981 \mathrm{~N}$ em região de molares. Isso gera falhas na cerâmica vítrea de cobertura que tem baixo valor de tenacidade e resistência à fratura [10]. Outros fatores também podem estar relacionados às altas taxas de lascamento, como incompatibilidade térmica entre os materiais, protocolo de resfriamento da cerâmica de recobrimento que pode induzir tensões residuais deletérias e baixa resistência de união entre a infraestrutura e a cerâmica de recobrimento [6]. Para resolver o problema clínico das altas taxas de lascamento foram desenvolvidas cerâmicas à base de zircônia translúcida para confecção de próteses monolíticas. A 3Y-TZP é opaca porque a luz que é transmitida no seu interior sofre espalhamento por várias fontes, como poros, inclusões, defeitos e contornos de grãos. Inicialmente, as estratégias utilizadas para melhorar a translucidez da 3Y-TZP foram o aumento da sua densidade, através da sinterização em temperaturas mais altas, e a redução da concentração da alumina utilizada como aditivo. A limitação dessas estratégias é que, para restaurações monolíticas com $1 \mathrm{~mm}$ ou mais de espessura, a 3 Y-TZP ainda é opaca $[6,11-13]$.

O cristal de zircônia tetragonal é birrefringente, ou seja, o índice de refração é diferente para diferentes direções cristalográficas, resultando em espalhamento do feixe de luz nos contornos de grãos e redução na transmitância. Então, outra estratégia utilizada é o desenvolvimento de cerâmica à base de zircônia cúbica completamente estabilizada. O cristal de zircônia cúbica é isotrópico, tem um índice de refração constante, e o feixe de luz não sofre esse espalhamento nos contornos dos grãos. O aumento do conteúdo de fase cúbica em cerâmicas à base de zircônia parcialmente estabilizada também pode ser obtido com o aumento do conteúdo de ítria (4 ou 5 mol\% de ítria; 4Y-PSZ e 5Y-PSZ). Entretanto, a zircônia cúbica não sofre transformação de fase sob tensão, o que compromete o comportamento mecânico dessas cerâmicas. A redução do tamanho de grão da 3 Y-TZP também é uma alternativa para obter um material translúcido e resistente à fratura, porém a fabricação de estruturas nanocristalinas de alta qualidade ainda é um desafio [1113]. No entanto, apesar de reduzir a possibilidade de fratura, eliminando a falha por lascamento, a degradação das propriedades mecânicas da zircônia é um motivo de preocupação. Isso porque a zircônia entra em contato direto com a saliva e com a carga mastigatória, o que pode aumentar a sua susceptibilidade ao crescimento subcrítico de trincas e contribuir para a transformação espontânea da fase tetragonal para monoclínica (low temperature degradation - LTD) [14]. Outro aspecto importante sobre restaurações monolíticas de zircônia é o desgaste dos elementos antagonistas, principalmente o esmalte dental, já que essas restaurações apresentam alta dureza e tenacidade à fratura. Ainda, estudos clínicos e laboratoriais têm mostrado que a zircônia apresenta alta resistência ao desgaste. Porém, quando comparada a outras cerâmicas e ao próprio esmalte dentário, existe divergência de resultados nas investigações [15-18]. Portanto, o objetivo deste estudo é verificar, através de uma revisão de literatura, o comportamento mecânico e clínico das próteses monolíticas à base de zircônia.

\section{MÉTODOS}

Foi feita uma busca na base de dados PubMed/Medline, durante o período de janeiro de 2010 a julho de 2017, utilizando as palavras-chave: zircônia (zirconia), monolítica (monolithic) e prótese (prosthesis). A seleção dos artigos ocorreu em duas etapas. A primeira etapa envolveu a seleção dos artigos laboratoriais. Os critérios de inclusão foram: métodos laboratoriais de caracterização do comportamento mecânico das cerâmicas; pelo menos um grupo experimental de cerâmica à base de zircônia para restauração monolítica; e corpos de prova de geometria simples ou na configuração de prótese. Foram excluídos os estudos laboratoriais de investigação das propriedades ópticas. A segunda etapa envolveu a seleção dos estudos clínicos. Foram incluídos estudos clínicos experimentais, estudos de coorte prospectivos e retrospectivos que avaliaram a longevidade de próteses monolíticas à base de zircônia. Não foram incluídos casos clínicos.

\section{RESULTADOS}

Foram selecionados treze estudos in vitro que estavam de acordo com os critérios de inclusão: cinco estudos avaliaram as propriedades mecânicas de corpos de prova de zircônia utilizando ensaios de resistência à flexão, ao lascamento e ao desgaste (Tabela I), e oito estudos avaliaram a carga de fratura e fadiga de próteses monolíticas (Tabela II). Cinco estudos clínicos de acompanhamento de próteses monolíticas à base de zircônia foram incluídos (Tabela III). Desses, três avaliaram apenas o desgaste das restaurações e dois avaliaram a sobrevivência ao longo do tempo.

\section{DISCUSSÃO}

Comportamento mecânico: os estudos de caracterização mecânica das cerâmicas à base de zircônia para próteses monolíticas indicam alta resistência ao lascamento [19] e alta resistência flexural $[4,5,20]$. Porém, existe variação nos valores das propriedades mecânicas entre os diferentes tipos de cerâmica à base de zircônia, dependendo da estratégia utilizada para alcançar uma maior translucidez [5]. Ainda, nenhum dos estudos citados acima avaliou as cerâmicas do tipo 4Y-PSZ e 5Y-PSZ, que têm maior conteúdo de zircônia cúbica e poderiam apresentar propriedades mecânicas 
Tabela I - Dados dos estudos in vitro: propriedades mecânicas - resistência flexural, ao lascamento e ao desgaste. [Table I - Data from in vitro studies: mechanical properties - flexural, chipping and wear resistance.]

\begin{tabular}{|c|c|c|c|c|}
\hline Ref. & Teste & Grupo & Resultado & Modo de falha \\
\hline [19] & $\begin{array}{c}\text { Resistência ao } \\
\text { lascamento e resistência } \\
\text { à flexão em três pontos }\end{array}$ & $\mathrm{ZM} \mathrm{e} \mathrm{DL}$ & $\begin{array}{l}\text { ZM apresentou maior resistência } \\
\text { flexural e ao lascamento }\end{array}$ & $\begin{array}{l}\text { Lascamento } \\
\text { e fratura } \\
\text { catastrófica }\end{array}$ \\
\hline [15] & $\begin{array}{l}\text { Resistência ao desgaste } \\
\quad(\text { pin-on-disk) }\end{array}$ & ZM, DL, RC e esmalte & $\begin{array}{c}\text { DL e esmalte desgastaram mais } \\
\text { o esmalte antagonista }\end{array}$ & - \\
\hline$[4]$ & $\begin{array}{l}\text { Resistência à flexão em } \\
\text { três pontos }\end{array}$ & ZM, técnica CAD-on (Z+DL) & $\begin{array}{c}\text { Resistência flexural foi } \\
\text { semelhante para os dois grupos; } \\
\text { a tenacidade à fratura foi maior } \\
\text { para o grupo CAD-on }\end{array}$ & $\begin{array}{c}\text { Fratura } \\
\text { catastrófica }\end{array}$ \\
\hline$[5]$ & $\begin{array}{c}\text { Resistência à flexão em } \\
\text { quatro pontos }\end{array}$ & $\begin{array}{l}\text { Quatro tipos de ZM translúcida } \\
\text { receberam envelhecimento em autoclave } \\
\text { por } 0,5,50,100,150 \text { e } 200 \mathrm{~h}\end{array}$ & $\begin{array}{l}\text { O efeito do envelhecimento foi } \\
\text { dependente do tipo de zircônia }\end{array}$ & $\begin{array}{c}\text { Fratura } \\
\text { catastrófica }\end{array}$ \\
\hline [20] & $\begin{array}{l}\text { Resistência à flexão } \\
\text { biaxial }\end{array}$ & $\begin{array}{l}\text { ZM sem desgaste, desgaste com pontas } \\
\text { diamantadas e pontas diamantadas } \\
\text { extrafinas; com e sem envelhecimento } \\
\text { em autoclave }\end{array}$ & $\begin{array}{l}\text { Não houve influência do tipo de } \\
\text { degaste e do envelhecimento em } \\
\text { autoclave na resistência flexural }\end{array}$ & $\begin{array}{c}\text { Fratura } \\
\text { catastrófica }\end{array}$ \\
\hline
\end{tabular}

Notas: ZM - zircônia na configuração monolítica; $D L$ - vitrocerâmica à base de dissilicato de lítio na configuração monolítica; RC - resina composta.

Tabela II - Dados dos estudos in vitro: ensaios de carga de fratura e fadiga de coroas monolíticas à base de zircônia. [Table II - Data from in vitro studies: fracture load and fatigue tests of monolithic zirconia crowns.]

\begin{tabular}{|c|c|c|c|c|}
\hline Ref. & Teste & Grupo & Resultado & Modo de falha \\
\hline [21] & Carga de fratura & $\begin{array}{c}\text { Coroas de ZM, ZC, DL, MC } \\
\text { (espessuras } 0,6,0,8,1,0,1,2 \mathrm{e} \\
1,5 \mathrm{~mm})\end{array}$ & $\begin{array}{l}\text { Coroas de ZM obtiveram os } \\
\text { maiores valores de carga de } \\
\text { fratura }\end{array}$ & $\begin{array}{c}\text { Fratura catastrófica para } \\
\text { ZM e DL; lascamento } \\
\text { para ZC e MC }\end{array}$ \\
\hline [14] & $\begin{array}{c}\text { Carga de fratura após } \\
\text { envelhecimento mecânico } \\
\left(80 \mathrm{~N}, 2,5.10^{6} \text { ciclos }\right)\end{array}$ & $\begin{array}{l}\text { Coroa de ZM polida; coroa de } \\
\mathrm{ZM} \text { com glaze; coroa de } \mathrm{ZC}\end{array}$ & $\begin{array}{l}\text { Coroas de ZM polidas ( } 3476 \\
\mathrm{~N}) \text { e com glaze }(3561 \mathrm{~N}) \\
\text { foram semelhantes e maiores } \\
\text { que } \mathrm{ZC}(2060 \mathrm{~N})\end{array}$ & $\begin{array}{l}\text { Fratura catastrófica para } \\
\text { ZM; lascamento para ZC }\end{array}$ \\
\hline$[22]$ & $\begin{array}{l}\text { Carga de fratura com e sem } \\
\text { envelhecimento mecânico } \\
\left(300 \mathrm{~N}, 2,4.10^{5} \text { ciclos }\right)\end{array}$ & $\begin{array}{c}\text { Coroas de } \mathrm{ZM} \text { após } \\
\text { envelhecimento em autoclave de } \\
0,10,50 \mathrm{e} 100 \mathrm{~h}\end{array}$ & $\begin{array}{l}\text { A carga de fratura diminuiu } \\
\text { após } 100 \text { h em autoclave; } \\
\text { envelhecimento mecânico não } \\
\text { teve influência }\end{array}$ & Fratura catastrófica \\
\hline [6] & $\begin{array}{c}\text { Carga de fratura após } \\
\text { envelhecimento térmico e } \\
\text { mecânico ( } 30 \text { e } 3000 \mathrm{~N}, 10^{4} \\
\text { ciclos) }\end{array}$ & $\begin{array}{c}\text { Coroas de ZM com alta e baixa } \\
\text { translucidez (espessura } 0,3,0,5 \\
0,7,1,0 \text { e } 1,5 \mathrm{~mm}) \text { e DL }(1,0 \mathrm{e} \\
1,5 \mathrm{~mm})\end{array}$ & $\begin{array}{l}\text { Não houve diferença na carga } \\
\text { de fratura entre ZM de alta } \\
\text { e baixa translucidez, sendo } \\
\text { superiores a DL }\end{array}$ & $\begin{array}{l}\text { Fratura catastrófica das } \\
\text { coroas e trincas }\end{array}$ \\
\hline$[23]$ & $\begin{array}{l}\text { Carga de fratura imediata; } \\
\text { após ciclagem térmica e } \\
\text { mecânica ( } 50 \mathrm{~N}, 1,2.10^{6} \\
\text { ciclos); após envelhecimento } \\
\text { em autoclave }\end{array}$ & $\begin{array}{l}\text { Coroas de } \mathrm{ZM} \text { com diferentes } \\
\text { tipos de preparos (sem ombro; } \\
0,4 \text { e } 0,8 \mathrm{~mm} \text { de chanfro) }\end{array}$ & $\begin{array}{l}\text { Houve interação entre os } \\
\text { fatores preparo e tipo de } \\
\text { envelhecimento }\end{array}$ & Fratura catastrófica \\
\hline$[24]$ & Carga de fratura & $\begin{array}{l}\text { Coroas de ZM cimentadas com } \\
\text { fosfato de zinco, ionômero } \\
\text { de vidro, cimento resinoso } \\
\text { convencional e autoadesivo }\end{array}$ & $\begin{array}{c}\text { Não houve diferença entre os } \\
\text { cimentos testados }\end{array}$ & Fratura catastrófica \\
\hline [25] & $\begin{array}{l}\text { Fadiga cíclica }(300,500 \text { e } \\
\left.800 \mathrm{~N}, 10^{6} \text { ciclos }\right)\end{array}$ & $\begin{array}{l}\text { Coroas de ZM (espessura } 0,4, \\
\quad 0,5,0,6,0,7 \text { e } 0,8 \mathrm{~mm})\end{array}$ & $\begin{array}{l}\text { Apenas as coroas com } \\
\text { espessura de } 0,7 \text { e } 0,8 \mathrm{~mm} \\
\text { resistiram a } 10^{6} \text { ciclos }\end{array}$ & Fratura catastrófica \\
\hline [26] & $\begin{array}{c}\text { Fadiga cíclica }\left(100 \mathrm{~N}, 2.10^{6}\right. \\
\text { ciclos })\end{array}$ & $\begin{array}{c}\text { Coroas de ZM, ZC e cobertura } \\
\text { parcial de porcelana }\end{array}$ & $\begin{array}{l}\text { Coroas multicamadas tiveram } \\
\text { alta susceptibilidade à fadiga }\end{array}$ & $\begin{array}{l}\text { Lascamento e trinca } \\
\text { da porcelana de } \\
\text { recobrimento }\end{array}$ \\
\hline
\end{tabular}

Notas: ZM - zircônia na configuração monolítica; ZC (controle) - zircônia na configuração bicamada, recoberta com cerâmica vítrea; DL - vitrocerâmica à base de dissilicato de lítio na configuração monolítica; $M C$ - metalocerâmica. 
Tabela III - Dados dos estudos clínicos.

[Table III - Data from clinical studies.]

\begin{tabular}{|c|c|c|c|c|c|}
\hline Ref. & $\begin{array}{l}\text { Tipo de } \\
\text { restauração }\end{array}$ & $\mathrm{n}$ & Tempo & Taxa de falhas & Modo de falha \\
\hline [16] & $\begin{array}{l}\text { Coroas de ZM } \\
\text { (molares) }\end{array}$ & 20 & 6 meses & $\begin{array}{l}\text { Coroas de ZM desgastaram mais } \\
\text { o antagonista }(33 \mu \mathrm{m}) \text { do que o } \\
\text { esmalte dentário }(10 \mu \mathrm{m})\end{array}$ & Não houve falhas \\
\hline$[18]$ & $\begin{array}{l}\text { Coroas de ZM } \\
\text { (molares) }\end{array}$ & 20 & $\begin{array}{c}\text { 6-12-24 } \\
\text { meses }\end{array}$ & $\begin{array}{l}\text { Coroas de ZM desgastaram mais } \\
\text { o antagonista }(46 \mu \mathrm{m}) \text { do que o } \\
\text { esmalte dentário }(19 \text { a } 26 \mu \mathrm{m})\end{array}$ & Não houve falhas \\
\hline [17] & $\begin{array}{l}\text { Coroas de ZM } \\
\text { (pré-molares e } \\
\text { molares) }\end{array}$ & 14 & 24 meses & $\begin{array}{c}\text { Não houve diferença no desgaste } \\
\text { de coroas de ZM e esmalte } \\
\text { dentário }\end{array}$ & Não houve falhas \\
\hline [27] & $\begin{array}{c}\text { Coroas de ZM } \\
\text { e ZC }\end{array}$ & $90 \mathrm{ZM} 72 \mathrm{ZC}$ & 36 meses & $\begin{array}{l}\text { Taxa de falha de } 1,5 \% \text { para ambos; } \\
\text { taxa de complicações de } 6,4 \% \\
(\mathrm{ZM}) \text { e } 4,5 \%(\mathrm{ZC}) \text {; taxa de fraturas } \\
\text { de } 0 \%(\mathrm{ZM}) \text { e } 1,5 \%(\mathrm{ZC})\end{array}$ & $\begin{array}{c}\text { Coroas de ZM não } \\
\text { apresentaram falhas, } \\
\text { apenas o dente pilar; } \\
\text { coroas de ZC apresentaram } \\
\text { lascamento }\end{array}$ \\
\hline [9] & $\begin{array}{c}\text { Coroas e PPFs } \\
\text { de ZM }\end{array}$ & $\begin{array}{c}39827 \text { restaurações, } \\
\text { sendo } 3731 \text { anteriores } \\
(1952 \text { coroas e } \\
1799 \text { PPFs) e } 36096 \\
\text { posteriores (29808 } \\
\text { coroas e } 6288 \text { PPFs) }\end{array}$ & 60 meses & $\begin{array}{c}\text { Taxa de falhas de } 0,97 \% \text { para } \\
\text { anterior, } 0,69 \% \text { para posterior } \\
\text { e } 0,71 \% \text { combinado; } 3,26 \% \text { de } \\
\text { fraturas em região anterior, } 2,42 \% \\
\text { para posterior e } 2,60 \% \text { combinado }\end{array}$ & Fratura catastrófica \\
\hline
\end{tabular}

Notas: ZM - zircônia na configuração monolítica; ZC (controle) - zircônia na configuração bicamada, recoberta com cerâmica vítrea.

inferiores [13]. O desenvolvimento de zircônias translúcidas para próteses monolíticas também tem como objetivo substituir as cerâmicas vítreas na confecção de restaurações para a região anterior, especialmente a vitrocerâmica à base de dissilicato de lítio (DL). A DL é amplamente utilizada de forma monolítica e apresenta uma boa combinação de propriedades mecânicas e ópticas que favorecem sua indicação para coroas unitárias anteriores e posteriores. Em comparação com a 3Y-TZP, a DL é mais estética, entretanto, apresenta menor resistência flexural, resistência ao lascamento e carga de fratura. Nos estudos, as falhas das coroas são geralmente catastróficas para ambas cerâmicas, mas coroas de DL também podem apresentar trincas $[6,19$, 21]. Em relação ao desgaste do antagonista, estudo in vitro indicou que a DL causa maior desgaste do esmalte dentário do que a 3Y-TZP [15].

Apesar da falta de evidências clínicas que comprovem a presença de degradação em baixas temperaturas (LTD) no ambiente oral e seu efeito na longevidade das próteses à base de zircônia, esse é um tópico de grande interesse nas investigações laboratoriais. O aumento da rugosidade da superfície e a proporção de transformação da fase tetragonal para monoclínica poderiam diminuir a resistência à fratura da 3Y-TZP pela formação de microtrincas, levando a falhas prematuras [23]. O envelhecimento em autoclave vem sendo utilizado para simular a LTD. Um estudo utilizou este método e encontrou relação entre a espessura da área transformada e a resistência flexural, sendo que o tipo de 3 Y-TZP com menor espessura transformada $(5 \mu \mathrm{m})$ obteve maior resistência flexural do que outro tipo de 3 Y-TZP que teve maior espessura $(60 \mu \mathrm{m})$ [5]. Já outro estudo avaliou a influência da rugosidade de superfície feita com pontas diamantadas e o envelhecimento em autoclave na resistência flexural da 3Y-TZP e não encontrou relação entre os fatores [20].É importante ressaltar que não existe uma padronização dos protocolos de envelhecimento por autoclave, o que dificulta a comparação entre os achados da literatura. Ainda, a susceptibilidade ao LTD depende da composição da cerâmica. Por exemplo, zircônias com maior conteúdo de fase cúbica são menos susceptíveis a LTD porque essa fase não sofre transformação [13].

Fadiga é o processo de falha que ocorre quando os materiais são sujeitos a tensões ou deformações por um período de tempo. Em boca, as próteses encontram-se em um ambiente que favorece o crescimento subcrítico de trincas e a falha por fadiga. Cerâmicas à base de zircônia têm alta resistência mecânica e dificilmente falham em poucos ciclos de fadiga, o que foi comprovado por estudos que não encontraram falhas após ciclagem com tempos reduzidos [14, 22, 23]. Um estudo usou carga constante e maior tempo de fadiga ( $100 \mathrm{~N}$ e 2 milhões de ciclos) e ainda assim não houve falhas das coroas monolíticas de 3Y-TZP. Já as coroas multicamadas à base de zircônia e metalocerâmica tiveram maior susceptibilidade à falha por fadiga e apresentaram lascamentos e trincas na porcelana de recobrimento [26]. Uma investigação relatou falhas de coroas monolíticas após 1 milhão de ciclos, mas a espessura das coroas que falharam era muito pequena $(0,4$ a $0,6 \mathrm{~mm})$ [25].

Preparos minimamente invasivos vêm sendo preconizados nos dias atuais, utilizando sobre estes próteses com espessura pequena, diminuindo a perda de tecido dental hígido. Em relação às coroas monolíticas à base de zircônia, 
estudos encontraram que uma espessura de no mínimo 0,7 $\mathrm{mm}$ deve ser usada para que seja suficientemente resistente às cargas impostas pela mastigação, tendo carga de fratura semelhante a coroas metalocerâmicas $[6,21,25]$. Um estudo avaliou a influência do preparo na carga de fratura de coroas monolíticas à base de zircônia e não encontrou diferença estatística entre os grupos sem ombro, 0,4 e $0,8 \mathrm{~mm}$ de chanfro [22]. Outro estudo avaliou o efeito do tipo de cimento na carga de fratura dessas coroas e não encontrou diferença entre fosfato de zinco, ionômero de vidro e cimento resinoso [24]. Cabe ressaltar que a maioria dos estudos utilizou a 3Y-TZP. Assim, é importante que o profissional compreenda a composição e microestrutura da cerâmica que irá utilizar para escolher os parâmetros de preparo e cimentação de acordo com o comportamento mecânico e óptico de cada material e considerando cada indicação clínica.

Comportamento clínico: foram encontrados dois estudos clínicos (um prospectivo e um retrospectivo) de avaliação da taxa de sobrevivência de próteses de zircônia monolíticas. $\mathrm{O}$ estudo prospectivo avaliou coroas à base de zircônia monolíticas e multicamadas com cobertura de porcelana. Após 36 meses, a taxa de sobrevivência foi de 98,5\% para ambos tipos de coroas. Os tipos de complicações encontrados para os dois tipos de prótese foram similares e envolveram no geral complicações biológicas, como cárie, problemas endodônticos, doença periodontal e fratura da raiz. Poucas complicações técnicas associadas às coroas, como perda de retenção e lascamento, foram observadas, o que indica bom comportamento mecânico para os dois tipos de tratamento reabilitador. Com relação à estética e satisfação dos dentistas e pacientes, os escores também foram semelhantes [27].

O estudo retrospectivo avaliou a taxa de falha de coroas e PPFs monolíticas à base de zircônia após 60 meses de uso clínico. Foram coletados os dados de dois laboratórios protéticos que recebiam restaurações de zircônia fraturadas. A taxa de falhas foi de 1,09\% em 5 anos (incluindo anterior e posterior), o que também sugere um bom desempenho clínico. Porém, ao analisar os dados separadamente, observou-se que restaurações em região anterior falharam menos do que na região posterior. Além disso, PPFs falharam mais do que coroas unitárias em ambas as regiões [9]. Três estudos clínicos prospectivos avaliaram o desgaste causado nos antagonistas por coroas monolíticas à base de zircônia. O desgaste das áreas de contato oclusal das coroas, do antagonista natural e de dois dentes naturais contralaterais foi mensurado usando réplicas e escaneamento com laser 3D. Em dois estudos do mesmo grupo, um de acompanhamento de 6 meses e outro de 2 anos, as coroas de zircônia desgastaram mais o antagonista do que o dente natural $[16,18]$. Já em outro estudo não houve diferença estatística entre o desgaste sofrido pelo esmalte dentário natural e coroas monolíticas à base de zircônia após dois anos de uso clínico [17]. A principal diferença entre os estudos foi o tipo de zircônia usada e a presença da camada de glaze. Nos estudos que desgastaram mais o antagonista foi usado o glaze e no estudo que teve menor desgaste não foi aplicado (apenas polimentos com pontas de borracha).
Próteses monolíticas à base de zircônia representam uma boa opção para reabilitar em região posterior de maxila e mandíbula, já que apresentam propriedades mecânicas mais do que suficientes para suportar as cargas dessa região. Esta modalidade de tratamento, que utiliza apenas um tipo de material restaurador, diminui significativamente as falhas por lascamento, aumentando a taxa de sobrevivência. Porém, deve-se considerar que diferentes estratégias vêm sendo utilizadas pelos fabricantes para obter um material mais translúcido. Essas modificações de composição e microestrutura da cerâmica devem melhorar as suas propriedades ópticas sem comprometer seu comportamento mecânico [13]. Além disso, estudos clínicos com maiores tempos de acompanhamento devem ser realizados.

\section{CONCLUSÕES}

Próteses monolíticas à base de zircônia representam uma boa opção de tratamento reabilitador porque vem resultando em boas taxas de sucesso clínico. Investigações laboratoriais mostram alta resistência à fratura, à fadiga e ao lascamento para 3Y-TZP utilizada em próteses monolíticas.

\section{REFERÊNCIAS}

[1] A. Della Bona, J.R. Kelly, J. Am. Dent. Assoc. 139 Suppl. (2008) 8S.

[2] M. Guazzato, M. Albakry, S.P. Ringer, M.V. Swain, Dent. Mater. 20 (2004) 441.

[3] M. Borba, M.D. de Araujo, K.A. Fukushima, H.N. Yoshimura, P.F. Cesar, J.A. Griggs, Dent. Mater. 27 (2011) 710.

[4] G.R. Basso, R.R. Moraes, M. Borba, J.A. Griggs, A. Della Bona, Dent. Mater. 31 (2015) 1453.

[5] B.D. Flinn, A.J. Raigrodski, L.A. Mancl, R. Toivola, T. Kuykendall. J. Prosthet. Dent. 117 (2017) 303.

[6] N. Nordahl, P.V. von Steyern, C. Larsson. J. Oral Sci. 57 (2015) 255.

[7] I. Sailer, N.A. Makarov, D.S. Thoma, M. Zwahlen, B.E. Pjetursson, Dent. Mater. 31 (2015) 603.

[8] B.E. Pjetursson, I. Sailer, N.A. Makarov, M. Zwahlen, D.S. Thoma, Dent. Mater. 31 (2015) 624.

[9] T.A. Sulaiman, A.A. Abdulmajeed, T.E. Donovan, L.F. Cooper, R. Walter, J. Prosthet. Dent. 116 (2016) 436.

[10] D. Augusti, G. Augusti, A. Borgonovo, M. Amato, D. Re, Case Rep. Dent. 2014 (2014) 629786.

[11] H. Tong, C.B. Tanaka, M.R. Kaizer, Y. Zhang, Ceram. Int. 42 (2016) 1077.

[12] Y. Zhang. Dent. Mater. 30 (2014) 1195.

[13] Y. Zhang, B.R. Lawn. J. Dent. Res. 97 (2017) 140.

[14] D.P. Lameira, W.A. Buarque e Silva, F. Andrade e Silva, G.M. De Souza, Biomed. Res. Int. 2015 (2015) 418641.

[15] J. Sripetchdanond, C. Leevailoj, J. Prosthet. Dent. 112 (2014) 1141.

[16] T. Stober, J.L. Bermejo, P. Rammelsberg, M. Schmitter, J. Oral Rehabil. 41 (2014) 314.

[17] U. Lohbauer, S. Reich. Clin. Oral Investig. 21 (2017) 
1165.

[18] T. Stober, J.L. Bermejo, F.S. Schwindling, M. Schmitter, J. Oral Rehabil. 43 (2016) 621.

[19] Y.Zhang, J.J. Lee, R. Srikanth, B.R. Lawn, Dent. Mater. 29 (2013) 1201.

[20] L.F. Guilardi, G.K. Pereira, A. Gundel, M.P. Rippe, L.F. Valandro. J. Mech. Behav. Biomed. Mater. 65 (2017) 849.

[21] T. Sun, S. Zhou, R. Lai, R. Liu, S. Ma, Z. Zhou, J. Mech. Behav. Biomed. Mater. 35 (2014) 93.

[22] K. Nakamura, A. Harada, T. Kanno, R. Inagaki, Y. Niwano, P. Milleding, U. Ortengren, J. Mech. Behav. Biomed. Mater. 47 (2015) 49.
[23] G. Mitov, Y. Anastassova-Yoshida, F.P. Nothdurft, C. von See, P. Pospiech. J. Adv. Prosthodont. 8 (2016) 30.

[24] K. Nakamura, M. Mouhat, J.M. Nergard, S.J. Laegreid, T. Kanno, P. Milleding, U. Ortengren, Acta Biomater. Odontol. Scand. 2 (2016) 12.

[25] T.H. Lan, P.H. Liu, M.M. Chou, H.E. Lee. J. Prosthet. Dent. 115 (2016) 76.

[26] G.F. Ramos, E.B. Monteiro, M.A. Bottino, Y.Zhang, R. Marques de Melo, Int. J. Period. Rest. Dent. 35 (2015) 843. [27] W. Bomicke, P. Rammelsberg, T. Stober, M. Schmitter. J. Esthet. Restor. Dent. 29 (2017) 22.

(Rec. 17/08/2017, Rev. 11/01/2018, 09/04/2018, Ac. 28/04/2018) 